2021, Instituto Mexicano de Tecnología del Agua

DOI: $10.24850 /$ j-tyca-2021-01-02

Artículos

\title{
Índice de calidad de agua en la microcuenca altoandina del río Chumbao, Andahuaylas, Apurímac, Perú
}

\section{Water quality index in the high-Andean micro-basin of the Chumbao River, Andahuaylas, Apurímac, Peru}

David Choque-Quispe ${ }^{1}$, ORCID: 0003-4002-7526

Carlos A. Ligarda-Samanez², ORCID: 0000-0001-7519-8355

Aydeé M. Solano-Reynoso33, ORCID: 0000-0002-1835-2210

Betsy S. Ramos-Pacheco4, ORCID: 0000-0002-0286-0632

Yadyra Quispe-Quispe5, ORCID: 0000-0002-5232-693X

Yudith Choque-Quispe ${ }^{6}$, ORCID: 0000-0002-3690-7267

Aydeé Kari-Ferro ${ }^{7}$

${ }^{1}$ Escuela de Ingeniería Agroindustrial, Universidad Nacional José María Arguedas, Andahuaylas, Perú, davidchoqueq@gmail.com

Escuela de Ingeniería Agroindustrial, Universidad Nacional José María Arguedas, Andahuaylas, Perú, carligarda@hotmail.com 
${ }^{3}$ Escuela de Ingeniería Ambiental, Universidad Tecnológica de los Andes, Andahuaylas, Perú, ayma_21@hotmail.com

4Escuela de Ingeniería Agroindustrial, Universidad Nacional José María Arguedas, Andahuaylas, Perú, bsramosp2@gmail.com

${ }^{5}$ Escuela de Ingeniería Ambiental, Universidad Alas Peruanas, Cusco, Perú, yandhy95@gmail.com

${ }^{6}$ Escuela de Ingeniería Ambiental, Universidad Tecnológica de los Andes, Cusco, Perú, yuditchoque@gmail.com

7Escuela de Ingeniería Agroecológica, Universidad Micaela Bastidas, Abancay, Perú, akariq14@hotmail.com

Autor para correspondencia: David Choque-Quispe, davidchoqueq@gmail.com

\section{Resumen}

El índice de calidad del agua (ICA) de un cuerpo hídrico indica el grado de contaminación para un determinado uso y está relacionado con las actividades antrópicas que suscitan alrededor. El objetivo fue determinar el ICA en la microcuenca altoandina del río Chumbao, Andahuaylas, Perú. El tramo de estudio estuvo comprendido entre la cabecera de cuenca ( $13^{\circ}$ 46' 42.5" S, 73 13' 50.0" O y $4295 \mathrm{~m}$ de altitud), y el último punto de muestreo ( $13^{\circ} 35^{\prime} 26.4^{\prime \prime} \mathrm{S}, 7^{\circ} 27^{\prime} 0.8^{\prime \prime}$ o y $2529 \mathrm{~m}$ de altitud), con 
pendiente media de $4 \%$. El ICA Dinius se evaluó considerando parámetros como nivel de oxígeno ( $\mathrm{OD}$ y $\mathrm{DBO}_{5}$ ); nivel de eutrofización $\left(\mathrm{NO}_{3}^{-}\right.$y $\left.\mathrm{PO}_{4}{ }^{3-}\right)$; características físicas (temperatura, turbidez, color y STD); sustancias disueltas (alcalinidad, dureza, $\mathrm{pH}$, conductividad y cloruros), y parámetros microbiológicos (E. coli y coliformes totales); se muestreó en temporada de avenidas (02 puntos lénticos y 08 lóticos). Los datos se recolectaron por triplicado y se analizaron a través de un ANOVA, test Tukey y correlación de Pearson al 5 \% de significancia. Se encontró que el uso predominante del agua del río es para agricultura, pastura y urbanización; el ICA está distribuido en tres categorías: Excelente (M1, M2, M3, M4 y M5), cercanos a la cabecera de la microcuenca y los puntos lénticos (lagunas de Pampahuasi y Paccoccocha); Aceptable (M6); y Excesivamente contaminado; los puntos M7 y M8 fuera de la zona urbana aguas abajo $(p$-value $<0.05)$ presentan buena correlación negativa con los parámetros de estudio. En conclusión, el agua en zonas de baja densidad poblacional es buena.

Palabras clave: altoandina, Dinius, ICA, microcuenca, río Chumbao.

\section{Abstract}

The water quality index (WQI) of a water body, indicates the degree of contamination for a given use, and it is related to the anthropic activities that they provoke around. The objective was to determine the WQI, in the high-Andean micro-basin of the Chumbao River, Andahuaylas, Peru. 
The section of study was included between the head of the basin $\left(13^{\circ} 46^{\prime}\right.$ 42.5" S, 73० 13' 50.0" W and $4295 \mathrm{~m}$ of altitude), and last sampling point ( $13^{\circ} 35^{\prime} 26.4^{\prime \prime} \mathrm{S}, 7^{\circ} 27^{\prime} 0.8^{\prime \prime} \mathrm{W}$ and $2529 \mathrm{~m}$ of altitude), with an average slope of $4 \%$. The ICA Dinius was evaluated considering parameters such as oxygen level ( $\mathrm{DO}$ and $\left.\mathrm{BOD}_{5}\right)$, eutrophication level $\left(\mathrm{NO}_{3}{ }^{-}\right.$and $\left.\mathrm{PO}_{4}{ }^{3-}\right)$, physical characteristics (temperature, turbidity, color and TDS), dissolved substances (alkalinity, hardness, $\mathrm{pH}$, conductivity and chlorides), and microbiological parameters (E. coli and total coliforms); it was sampled during the avenues season ( 02 lentic points and 08 lotics). The data were collected in triplicate, and analyzed through an ANOVA, Tukey test and Pearson's correlation at $5 \%$ significance. It was found that the predominant use of river water is for agriculture, pasture and urbanization; the WQI is divided into three categories Excellent (M1, M2, M3, M4 and M5) close to the head of the micro-basin and the lentic points (Pampahuasi and Paccoccocha lagoons); Acceptable (M6), and excessively contaminated; points M7 and M8 outside the urban area downstream ( $p$-value $<0.05$ ) present a good negative correlation with the study parameters. In conclusion, the water in areas of low population density is good.

Keywords: High-Andean, Dinius, ICA, micro-basin, Chumbao River.

Recibido: 19/02/2019

Aceptado: $18 / 04 / 2020$ 
Tecnología y

Ciencias $\stackrel{\unlhd}{\unlhd}$ gua
2021, Instituto Mexicano de Tecnología del Agua

Open Access bajo la licencia CC BY-NC-SA 4.0

(https://creativecommons.org/licenses/by-nc-sa/4.0/)

\section{Introducción}

Los ríos pueden contaminarse en su trayecto desde el nacimiento hasta su desembocadura, sobre todo aquellos que trascurren por zonas urbanizadas y transportan niveles excesivos de nitrógeno, fósforo y materia orgánica, que se dispersan a través de las aguas de escorrentía pluvial (Bhatti \& Latif, 2011); por otra parte, la mayoría de los ríos se ven afectados por descargas de aguas usadas sin tratar, provenientes de vertederos clandestinos, rellenos sanitarios, y desechos industriales líquidos y sólidos (Mophin-Kani \& Murugesan, 2011), lo que genera problemas ambientales y en la calidad de los cuerpos de agua (Blume et al., 2010; Benvenuti, Kieling-Rubio, Klauck, \& Rodrigues, 2015); el río Chumbao no es ajeno a esta realidad.

Asimismo, las actividades antrópicas circundantes a la cuenca de un río, como ganadería, agricultura y extracción minera, generan impactos negativos sobre la calidad del agua en un río, sobre todo si la capacidad de autodepuración de cuerpo de agua es baja, o si los componentes 
contaminantes no son biodegradables, o presentan trazas de metales disueltos y material inorgánico.

La calidad del agua se evalúa a través de parámetros fisicoquímicos, como $\mathrm{pH}$, oxígeno disuelto, demanda bioquímica de oxígeno y otros; además, con indicadores microbiológicos como el E. coli, que muestran la actividad antrópica de una región (Froehner, Machado, Botelho, \& Cordova-Da-Rosa, 2010; Dhawde et al., 2018; Abbas \& Hassan, 2018).

Horton (1965) propuso una forma de cualificar el estado de un cuerpo hídrico a través del índice de calidad de agua (ICA); fue pionero en desarrollar una metodología unificada para su cálculo; sin embargo, el desarrollo e implementación de un ICA de manera formal y demostrada lo realizaron Brown, Macclelland, Deininger y Tozer (1970), con el apoyo de la Fundación Nacional de Saneamiento (NSF), denominado índice NSF y Dinius (Dinus, 1987), basados en parámetros fisicoquímicos y microbiológicos; consideraron la estructura del índice de Horton y el método Delphi para definir parámetros, pesos ponderados, subíndices y clasificación a ser empleados en el cálculo (Prakirake, Chaiprasert, \& Tripetchkul, 2009; Bharti \& Katyal, 2011).

La ciudad de Andahuaylas en el Perú y el río Chumbao comparten el mismo espacio en la microcuenca, sin embargo, la ciudad no ha sido capaz de establecer una dinámica positiva de coexistencia con el río: ha cortado sus fluidos naturales, afloramientos y bosques ribereños; utiliza sus aguas para actividades agrícolas en temporada de estiaje, 
produciendo diversos vegetales que son comercializados en el mercado local, lo cual podría provocar problemas de salud en la población (AguirreMartínez, André, Gagné, \& Martín-Díaz, 2018). En ese sentido, el objetivo del trabajo fue evaluar el índice de calidad de agua en la microcuenca altoandina del río Chumbao, cuyos resultados permitirán la priorización de proyectos de saneamiento ambiental en la zona de impacto.

\section{Materiales y métodos}

\section{Descripción del área de estudio}

El estudio se realizó en temporada de avenidas en el mes de marzo de 2018, en la microcuenca del río Chumbao (Figura 1), ubicada en la provincia de Andahuaylas, Apurímac, Perú, en el tramo comprendido entre la cabecera de cuenca $\left(13^{\circ} 46^{\prime} 42.5^{\prime \prime} \mathrm{S}, 7^{\circ} 13^{\prime} 50.0^{\prime \prime}\right.$ O y 4295 m 
Tecnología y

\section{Ciencias $\stackrel{\Im}{\unlhd}$ Agua}

2021, Instituto Mexicano de Tecnología del Agua

Open Access bajo la licencia CC BY-NC-SA 4.0

(https://creativecommons.org/licenses/by-nc-sa/4.0/)

de altitud), y el último punto de muestreo ( $13^{\circ} 35^{\prime} 26.4^{\prime \prime} \mathrm{S}, 7^{\circ} 27^{\prime} 0.8^{\prime \prime}$ O y 2529 m de altitud); la microcuenca presenta un clima Cwb de acuerdo con Köppen's, con temporadas marcadas, en avenidas con precipitaciones intensas entre octubre y marzo (de 500 a 1000 mm/año), y temperaturas de 5 a $23^{\circ} \mathrm{C}$, humedad relativa media de $55 \%$. En la Figura 2 se muestra la distribución de lluvia durante el periodo experimental.
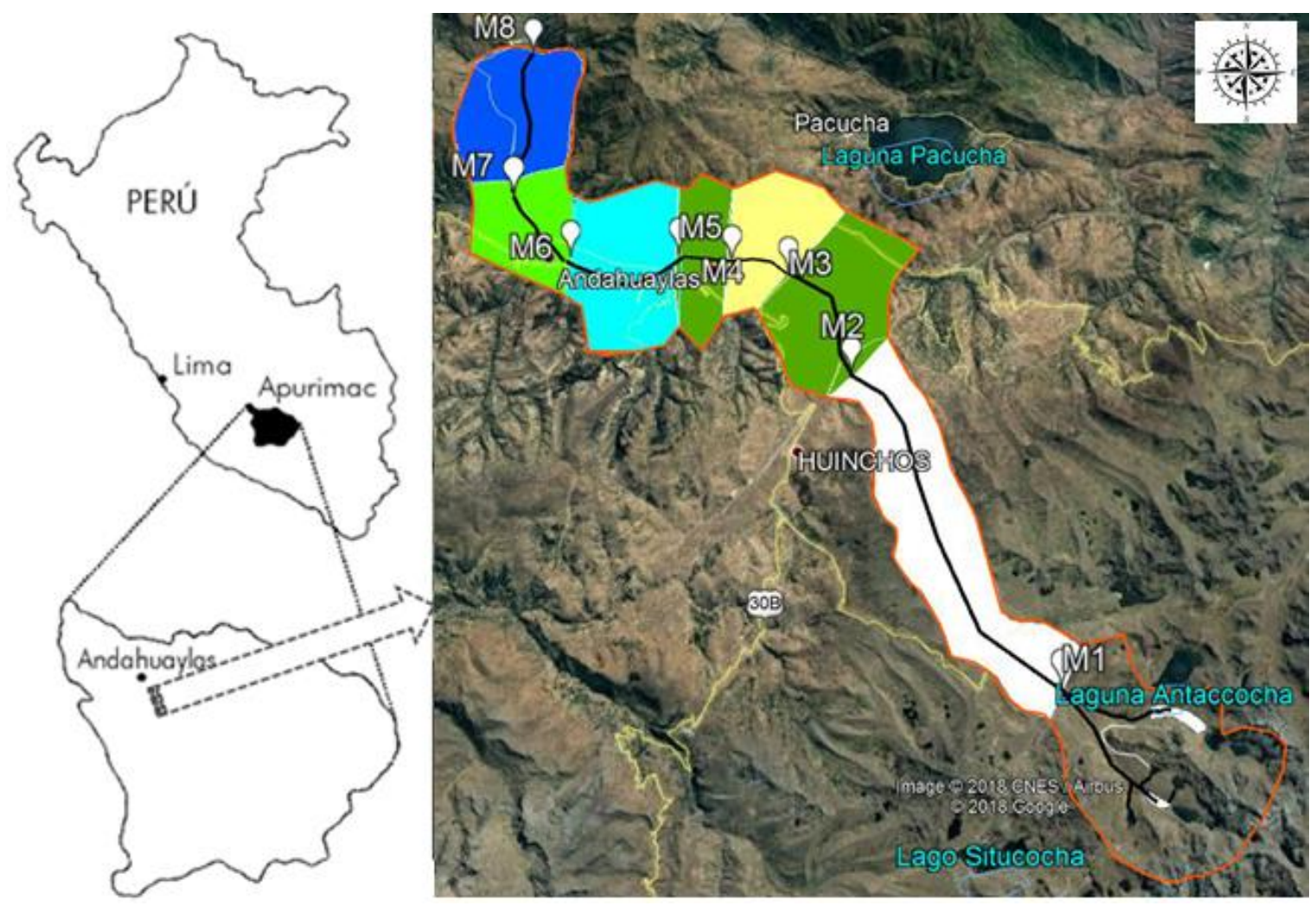

Figura 1. Microcuenca del río Chumbao. 
Teçnología y

Ciencias $₫$ Agua
2021, Instituto Mexicano de Tecnología del Agua

Open Access bajo la licencia CC BY-NC-SA 4.0

(https://creativecommons.org/licenses/by-nc-sa/4.0/)

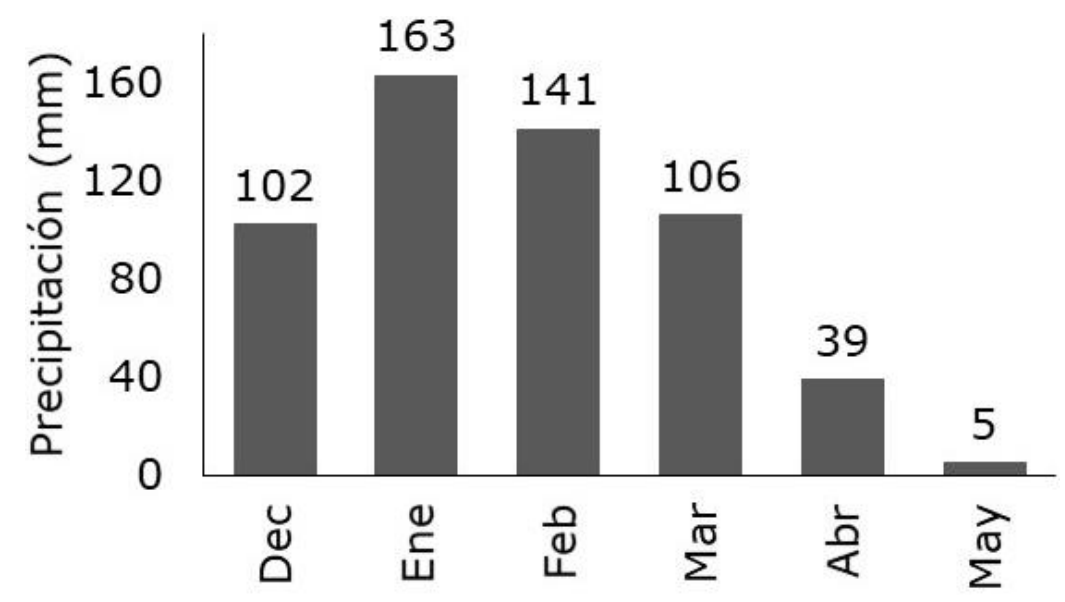

Figura 2. Precipitaciones entre diciembre y mayo de 2018.

\section{Puntos de muestreo}

Se identificaron 08 puntos lóticos a lo largo del río Chumbao y 02 puntos lénticos en la cabecera de la microcuenca, correspondientes a las lagunas Pampahuasi y Paccoccocha (Figura 3). 
2021, Instituto Mexicano de Tecnología del Agua

Tecnología y

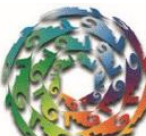

Ciencias $₫$ Agua

Open Access bajo la licencia CC BY-NC-SA 4.0

(https://creativecommons.org/licenses/by-nc-sa/4.0/)

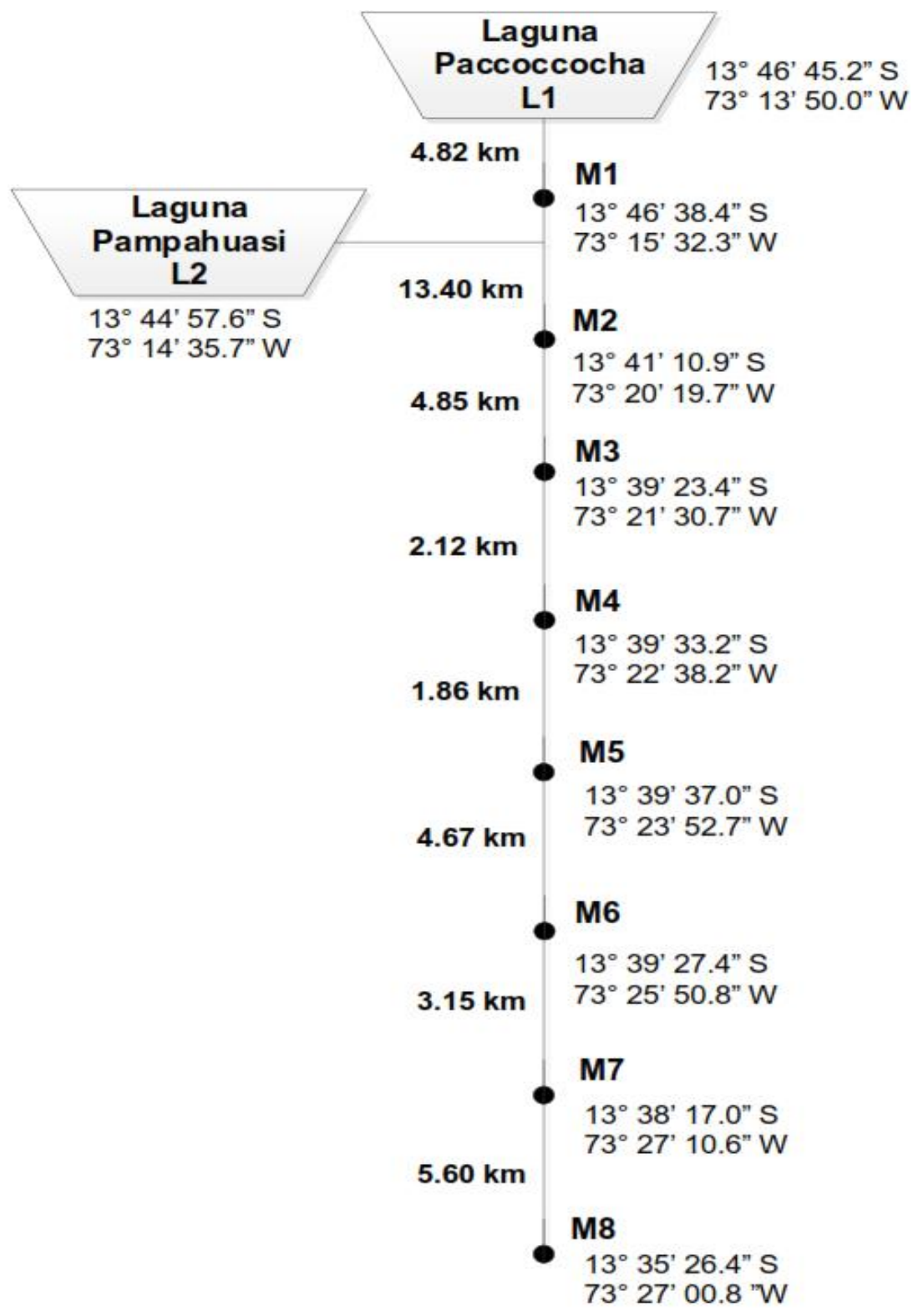

Figura 3. Puntos de muestreo en la microcuenca del río Chumbao. 


\section{Esquema metodológico}

El estudio se realizó de acuerdo con el esquema metodológico de campo y laboratorio, que se muestra en la Figura 4.

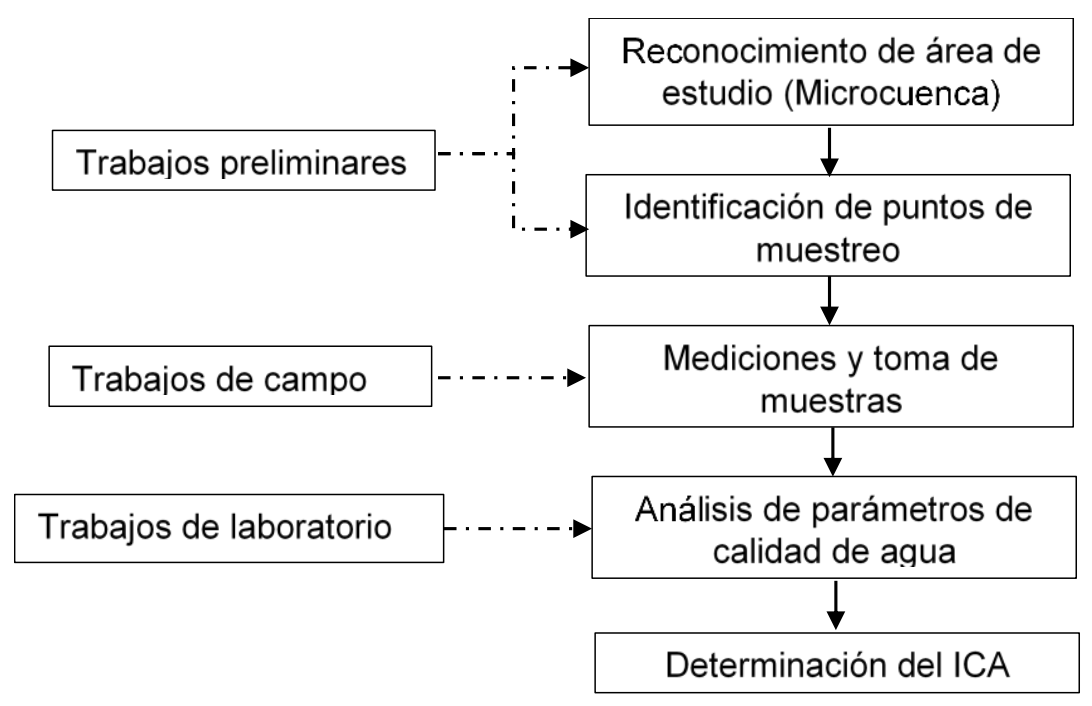

Figura 4. Flujograma metodológico de trabajo. 


\section{Indicadores fisicoquímicos y microbiológicos}

Los indicadores como la demanda bioquímica de oxígeno (DBO), nitratos, fosfatos, sólidos totales disueltos (STD), color, alcalinidad y dureza se determinaron siguiendo la metodología estándar para el análisis de agua y aguas residuales propuesta por la APHA (2012); mientras que el oxígeno disuelto (OD), temperatura, turbidez, $\mathrm{pH}$ y conductividad se determinaron considerando el manual de uso de multiparámetro HI 9829, previamente calibrado; por otra parte, los coliformes totales y $E$. coli se cuantificaron a través del uso de Placas Petrifilm 3M.

\section{Determinación de ICA}


Se evaluó el ICA según Dinius, considerando los parámetros ponderados mostrados en la Tabla 1, basados en la media geométrica (Ecuación (1)). La categorización de la calidad de los ICA se detalla en la Tabla 2:

$I C A_{m}=\prod_{i=1}^{n} I_{i}^{W_{i}}$

Donde $I C A_{m}$ es el índice de calidad de agua; $I_{i}$, el subíndice del parámetro $i$, y $W_{i}$ es el factor de ponderación para el subíndice $i$.

Tabla 1. Ponderación de indicadores para el ICA.

\begin{tabular}{|c|c|c|c|c|}
\hline \multirow{2}{*}{ Parámetro } & \multirow{2}{*}{ Unidad } & & \multicolumn{2}{|c|}{ Índice de calidad } \\
\hline & & & NSF & Dinius \\
\hline OD & $\mathrm{mg} \mathrm{O}_{2} / \mathrm{l}$ & $\mathrm{P} 1$ & 0.170 & 0.109 \\
\hline DBO & $\mathrm{mg} \mathrm{O}_{2} / \mathrm{l}$ & P2 & 0.110 & 0.097 \\
\hline Nitratos & $\mathrm{mg} / \mathrm{l}$ & P3 & 0.100 & 0.090 \\
\hline Fosfatos & $\mathrm{mg} / \mathrm{l}$ & P4 & 0.100 & --- \\
\hline Temperatura & ${ }^{\circ} \mathrm{C}$ & P5 & 0.100 & 0.077 \\
\hline Turbiedad & NTU & P6 & 0.080 & --- \\
\hline STD & $\mathrm{mg} / \mathrm{l}$ & P7 & 0.070 & --- \\
\hline
\end{tabular}


Tecnología y

Ciencias $₫$ Agua
2021, Instituto Mexicano de Tecnología del Agua

Open Access bajo la licencia CC BY-NC-SA 4.0

(https://creativecommons.org/licenses/by-nc-sa/4.0/)
Color

Alcalinidad

Dureza

$\mathrm{pH}$

Conductividad

Cloruros

E. coli

Coliformes totales
PCU

$\mathrm{mg} \mathrm{CaCO}_{3} / \mathrm{I}$

$\mathrm{mg} \mathrm{CaCO}_{3} / \mathrm{I}$

$\log \left[\mathrm{H}^{+}\right]$

$\mu \mathrm{S} / \mathrm{cm}$

$\mathrm{mg} / \mathrm{l}$

$\mathrm{NMP} / 100 \mathrm{ml}$

$\mathrm{NMP} / 100 \mathrm{ml}$
P8

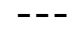

P9

$---$

P10

$---$

P11

0.110

P12

$--$

P13

$---$

P14 0.160

0.116

P15
0.074

0.063

0.063

0.065

0.077

0.079
0.090

Donde $\mathrm{Pi}=$ parámetros.

Tabla 2. Categorización Dinius para el ICA.

\begin{tabular}{lcc}
\hline \multicolumn{1}{c}{ Calidad } & Rango & Color \\
\hline Excelente & $70-100$ & Azul \\
Aceptable & $60-70$ & Celeste \\
Levemente contaminada & $50-60$ & Verde \\
Contaminada & $40-50$ & Amarillo \\
Fuertemente contaminada & $30-40$ & Naranja \\
Excesivamente contaminada & $0-30$ & Rojo \\
\hline
\end{tabular}


2021, Instituto Mexicano de Tecnología del Agua

Tecnología y

Open Access bajo la licencia CC BY-NC-SA 4.0

(https://creativecommons.org/licenses/by-nc-sa/4.0/)

Los parámetros fisicoquímicos estudiados fueron comparados con el agua de Categoría 4: conservación del ambiente acuático para ríos de la sierra, propuesto en el D.S. Nº 004-2017-MINAM, Perú (MINAM, 2017).

\section{Análisis estadísticos}

Los datos se recolectaron por triplicado, y se realizó un análisis de varianza (ANOVA) y comparación múltiple Tukey; asimismo, se determinó el coeficiente de correlación de Pearson a un nivel de significancia del 5 $\%$.

\section{Resultados y discusión}


En la Tabla 3 se presenta la descripción de la zona de estudio, el $23.6 \%$ se considera como cuenca colectora y, en menor porcentaje, el suelo es destinado para la urbanización e industria urbana; los puntos de muestreo lénticos y lóticos del río Chumbao están distribuidos a través de un recorrido del agua de $40.47 \mathrm{~km}$ (Figura 2), presentando tres tramos de pendientes pronunciadas, en el tramo del punto M1 a M3 $(18.25 \mathrm{~km}) \mathrm{la}$ pendiente es de $5.4 \%$, de M3 a M7 $(11.8 \mathrm{~km})$ la pendiente disminuye hasta un valor promedio de $2.2 \%$, y un último tramo de $5.60 \mathrm{~km}$ presenta una pendiente de $4.3 \%$, las pendientes pronunciadas incrementan la velocidad del agua y consecuentemente permiten una mejor aireación (Rivera, 2011), lo que permite mejorar el potencial de autopurificación del agua en un río (Feria-Díaz, Náder-Salgado, \& Meza-Pérez, 2017).

Tabla 3. Uso actual del suelo en la microcuenca del río Chumbao.

\begin{tabular}{ccccc}
\hline Área de influencia & Altitud $(\mathbf{m})$ & Uso actual & $\begin{array}{c}\text { Área } \\
\left.\mathbf{( k m}^{\mathbf{2}}\right)\end{array}$ & \% \\
\hline $\begin{array}{c}\text { Laguna } \\
\text { Pampahuasi-L1 }\end{array}$ & 4212 & Reservorio & 0.62 & --- \\
\hline $\begin{array}{c}\text { Laguna } \\
\text { Paccoccocha-L2 }\end{array}$ & 4274 & Reservorio & 0.17 & -- \\
\hline
\end{tabular}


Tecnología y

Ciencias $₫$ Agua
2021, Instituto Mexicano de Tecnología del Agua

Open Access bajo la licencia CC BY-NC-SA 4.0

(https://creativecommons.org/licenses/by-nc-sa/4.0/)

\begin{tabular}{|c|c|c|c|c|}
\hline M1 & 4081 & Cuenca colectora & 35.3 & 23.6 \\
\hline M1-M2 & $4081-3198$ & $\begin{array}{c}\text { Agricultura, pastura y } \\
\text { cuenca colectora }\end{array}$ & 14.8 & 9.9 \\
\hline M2-M3 & $3198-2992$ & $\begin{array}{c}\text { Agricultura limitada, } \\
\text { pastura limitada y } \\
\text { urbanización }\end{array}$ & 21.7 & 14.5 \\
\hline M3-M4 & $2992-2922$ & Urbanización e industria & 13.3 & 8.9 \\
\hline M4-M5 & $2922-2875$ & urbana limitada & 10.2 & 6.8 \\
\hline M5-M6 & $2875-2817$ & $\begin{array}{l}\text { Agricultura limitada, } \\
\text { pastura limitada y }\end{array}$ & 21.2 & 14.2 \\
\hline M6-M7 & $2817-2767$ & urbanización & 12.8 & 8.6 \\
\hline M7-M8 & $2767-2529$ & $\begin{array}{l}\text { Agricultura, pastura y } \\
\text { urbanización limitada }\end{array}$ & 20.0 & 13.4 \\
\hline
\end{tabular}

En la Tabla 4 se aprecia que el nivel de oxígeno disuelto se incrementa ligeramente en los puntos de muestreo conforme discurre aguas abajo ( $p$-value < 0.05), encontrándose valores mayores a lo recomendado por los estándares de calidad ambiental para el agua (ECA)Perú (MINAM, 2017); esto se debe sobre todo a la disminución de la altitud (Jacobsen \& Brodersen, 2008; Torres, Cruz, Patiño, Escobar, \& Pérez, 2010); alta turbulencia causada por la pendiente del río, distribución de las comunidades animales y vegetales (Sanders, 2002), 
Tecnología y

Ciencias $\stackrel{\varpi}{\triangleleft}$ Agua
2021, Instituto Mexicano de Tecnología del Agua

Open Access bajo la licencia CC BY-NC-SA 4.0

(https://creativecommons.org/licenses/by-nc-sa/4.0/)

incrementándose en variedades, desde ichu por encima de 3000 msnm, y por debajo a eucaliptos, plantas y árboles propios de la zona.

Tabla 4. Valores de indicadores fisicoquímicos y microbiológicos.

\begin{tabular}{|c|c|c|c|c|c|c|c|c|c|c|c|c|c|c|c|}
\hline & \multicolumn{3}{|c|}{ M1 } & \multicolumn{3}{|c|}{ M2 } & \multicolumn{3}{|c|}{ M3 } & \multicolumn{3}{|c|}{ M4 } & \multicolumn{3}{|c|}{ M5 } \\
\hline & $\overline{\bar{x}}$ & \pm & $\mathrm{s}$ & $\bar{x}$ & \pm & $\mathrm{s}$ & $\bar{x}$ & \pm & $\mathrm{s}$ & $\bar{x}$ & \pm & $\mathrm{S}$ & $\bar{x}$ & \pm & $\mathrm{s}$ \\
\hline P1 & 6.97 & \pm & 0.07 & 7.33 & \pm & $\begin{array}{l}0.1 \\
1\end{array}$ & 7.55 & \pm & 0.12 & 7.34 & \pm & 0.11 & 7.37 & \pm & $\begin{array}{l}0.0 \\
7\end{array}$ \\
\hline P2 & 1.26 & \pm & 0.13 & 2.67 & \pm & $\begin{array}{l}0.2 \\
8\end{array}$ & 2.39 & \pm & 0.25 & 7.70 & \pm & 0.81 & 7.72 & \pm & $\begin{array}{l}0.7 \\
0\end{array}$ \\
\hline P3 & 0.00 & \pm & 0.00 & 0.00 & \pm & $\begin{array}{l}0.0 \\
0\end{array}$ & 0.00 & \pm & 0.00 & 0.07 & \pm & 0.06 & 0.13 & \pm & $\begin{array}{l}0.0 \\
6\end{array}$ \\
\hline P4 & 0.07 & \pm & 0.01 & 0.04 & \pm & $\begin{array}{l}0.0 \\
1\end{array}$ & 0.06 & \pm & 0.01 & 0.15 & \pm & 0.01 & 0.11 & \pm & $\begin{array}{l}0.0 \\
1\end{array}$ \\
\hline P5 & 10.99 & \pm & 0.11 & 13.43 & \pm & $\begin{array}{l}0.2 \\
0\end{array}$ & 13.65 & \pm & 0.21 & 13.88 & \pm & 0.21 & 14.09 & \pm & $\begin{array}{l}0.2 \\
1\end{array}$ \\
\hline P6 & 42.50 & \pm & 0.43 & 36.00 & \pm & $\begin{array}{l}0.3 \\
6\end{array}$ & 36.82 & \pm & 0.56 & 50.87 & \pm & 0.77 & 45.25 & \pm & $\begin{array}{l}0.6 \\
9\end{array}$ \\
\hline P7 & 14.02 & t & 0.39 & 25.04 & \pm & $\begin{array}{l}0.6 \\
9\end{array}$ & 29.05 & \pm & 0.80 & 42.07 & \pm & 1.16 & 53.10 & \pm & $\begin{array}{l}1.4 \\
5\end{array}$ \\
\hline P8 & 29.05 & \pm & 0.80 & 24.04 & \pm & $\begin{array}{l}0.6 \\
6\end{array}$ & 14.02 & \pm & 0.39 & 37.06 & \pm & 1.02 & 27.05 & \pm & $\begin{array}{l}0.7 \\
4\end{array}$ \\
\hline P9 & 0.00 & \pm & 0.00 & 0.00 & \pm & $\begin{array}{l}0.0 \\
0\end{array}$ & 0.00 & \pm & 0.00 & 0.00 & \pm & 0.00 & 12.07 & \pm & $\begin{array}{l}1.1 \\
0\end{array}$ \\
\hline
\end{tabular}


Tecnología y

Ciencias $₫$ Agua
2021, Instituto Mexicano de Tecnología del Agua

Open Access bajo la licencia CC BY-NC-SA 4.0

(https://creativecommons.org/licenses/by-nc-sa/4.0/)

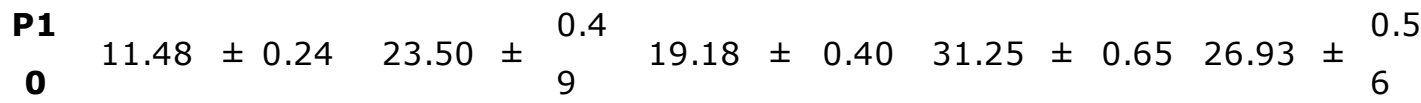

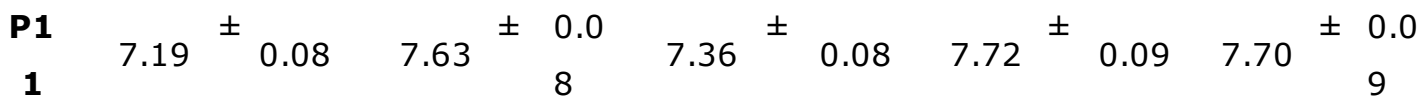

$$
\begin{aligned}
& \begin{array}{c}
\text { P1 } \\
2
\end{array} 29.33 \pm 1.53 \quad 51.00 \pm \begin{array}{l}
2.6 \\
5
\end{array} 60.00 \pm 3.6185 .67 \pm 4.73 \quad 108.0 \pm \begin{array}{l}
6.2 \\
4
\end{array}
\end{aligned}
$$

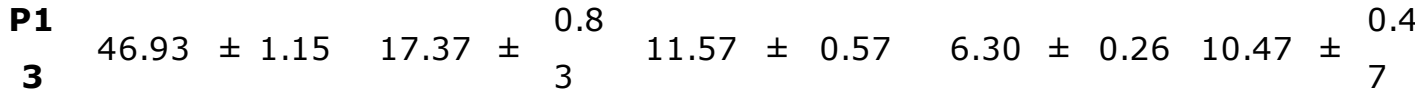

$$
\begin{aligned}
& \begin{array}{llllll}
\text { P1 } & 0.00 & 0.00 & 0.00 & 692.00 & 462.00
\end{array} \\
& \begin{array}{crrrrr}
\text { P1 } & 15.70 & 15.70 & 63.60 & 1100.0 & 1690.0 \\
\hline 5 & & &
\end{array}
\end{aligned}
$$

\begin{tabular}{|c|c|c|c|c|c|c|c|c|c|c|c|c|c|c|c|c|}
\hline & \multicolumn{3}{|c|}{ M6 } & \multicolumn{3}{|c|}{ M7 } & \multicolumn{3}{|c|}{ M8 } & \multicolumn{3}{|c|}{ L1 } & \multicolumn{3}{|c|}{ L2 } & \multirow[t]{2}{*}{ p-value } \\
\hline & $\overline{\bar{x}}$ & \pm & $\mathrm{s}$ & $\overline{\bar{x}}$ & \pm & $\mathrm{s}$ & $\bar{x}$ & \pm & $\mathrm{s}$ & $\overline{\bar{x}}$ & \pm & $\mathrm{s}$ & $\bar{x}$ & \pm & $\mathrm{s}$ & \\
\hline P1 & 7.15 & \pm & 0.11 & 7.30 & \pm & $\begin{array}{l}0.1 \\
1\end{array}$ & 7.86 & \pm & 0.08 & 6.09 & \pm & 0.06 & 5.92 & \pm & $\begin{array}{l}0.0 \\
6\end{array}$ & $>0.05$ \\
\hline P2 & 9.70 & \pm & 0.88 & 14.28 & \pm & $\begin{array}{l}1.3 \\
0\end{array}$ & 15.26 & \pm & 1.39 & 0.05 & \pm & 0.00 & 0.41 & \pm & $\begin{array}{l}0.0 \\
4\end{array}$ & $>0.05$ \\
\hline P3 & 0.47 & \pm & 0.12 & 0.50 & \pm & $\begin{array}{l}0.1 \\
0\end{array}$ & 0.97 & \pm & 0.15 & 0.00 & \pm & 0.00 & 0.00 & \pm & $\begin{array}{l}0.0 \\
0\end{array}$ & $>0.05$ \\
\hline P4 & 0.18 & \pm & 0.01 & 0.43 & \pm & $\begin{array}{l}0.0 \\
2\end{array}$ & 0.32 & \pm & 0.02 & 0.00 & \pm & 0.00 & 0.00 & \pm & $\begin{array}{l}0.0 \\
0\end{array}$ & $>0.05$ \\
\hline P5 & 14.59 & & 0.22 & 15.08 & \pm & $\begin{array}{l}0.2 \\
3\end{array}$ & 15.86 & \pm & 0.24 & 9.80 & \pm & 0.15 & 10.02 & \pm & $\begin{array}{l}0.1 \\
5\end{array}$ & $>0.05$ \\
\hline P6 & 91.00 & \pm & 1.38 & 112.36 & \pm & $\begin{array}{l}0.9 \\
2\end{array}$ & 140.5 & \pm & 1.16 & 1.43 & \pm & 0.21 & 0.47 & \pm & $\begin{array}{l}0.4 \\
2\end{array}$ & $>0.05$ \\
\hline P7 & 75.13 & \pm & 0.96 & 101.87 & \pm & $\begin{array}{l}1.3 \\
3\end{array}$ & 152.5 & \pm & 1.80 & 39.07 & \pm & 1.10 & 12.02 & \pm & $\begin{array}{l}0.3 \\
3\end{array}$ & $>0.05$ \\
\hline
\end{tabular}




\section{1, Instituto Mexicano de Tecnología del Agua}

Tecnología y

Open Access bajo la licencia CC BY-NC-SA 4.0

(https://creativecommons.org/licenses/by-nc-sa/4.0/)

\begin{tabular}{|c|c|c|c|c|c|c|c|c|c|c|c|c|c|c|}
\hline P8 & 35.06 & \pm 0.96 & 40.33 & \pm & $\begin{array}{l}0.7 \\
6\end{array}$ & 18.03 & \pm & 0.50 & 30.05 & \pm & 0.83 & $12.02 \pm$ & $\begin{array}{l}0.3 \\
3\end{array}$ & $>0.05$ \\
\hline P9 & 20.03 & \pm 0.55 & 40.07 & \pm & $\begin{array}{l}1.1 \\
0\end{array}$ & 55.09 & \pm & 1.51 & 0.00 & \pm & 0.00 & $0.00 \pm$ & $\begin{array}{l}0.0 \\
0\end{array}$ & $>0.05$ \\
\hline $\begin{array}{c}\mathbf{P 1} \\
\mathbf{0}\end{array}$ & 53.35 & \pm 1.10 & 68.09 & \pm & $\begin{array}{l}1.4 \\
1\end{array}$ & 54.88 & \pm & 1.13 & 17.07 & \pm & 0.35 & $8.84 \pm$ & $\begin{array}{l}0.1 \\
8\end{array}$ & $>0.05$ \\
\hline $\begin{array}{c}\text { P1 } \\
1\end{array}$ & 7.82 & ${ }^{ \pm} 0.09$ & 7.77 & \pm & $\begin{array}{l}0.0 \\
9\end{array}$ & 8.05 & \pm & 0.09 & 6.97 & \pm & 0.07 & $7.11^{ \pm}$ & $\begin{array}{l}0.0 \\
8\end{array}$ & $>0.05$ \\
\hline $\begin{array}{c}\mathbf{P 1} \\
\mathbf{2}\end{array}$ & 155.0 & \pm 8.89 & 204.00 & \pm & $\begin{array}{l}4.5 \\
8\end{array}$ & 302.3 & \pm & 8.50 & 79.67 & \pm & 3.06 & $25.33 \pm$ & $\begin{array}{l}1.5 \\
3\end{array}$ & $>0.05$ \\
\hline $\begin{array}{c}\mathbf{P 1} \\
\mathbf{3}\end{array}$ & 11.67 & \pm 0.57 & 16.63 & \pm & $\begin{array}{l}0.7 \\
8\end{array}$ & 30.07 & \pm & 0.96 & 38.17 & \pm & 1.82 & $40.13 \pm$ & $\begin{array}{l}1.9 \\
3\end{array}$ & $>0.05$ \\
\hline $\begin{array}{c}\text { P1 } \\
4\end{array}$ & 11 & 00.0 & 27 & 16.0 & & 21 & 59.0 & & & 1.00 & & 0.00 & & \\
\hline $\begin{array}{l}\mathbf{P 1} \\
5\end{array}$ & 27 & 16.0 & 32 & 82.0 & & 23 & 305.0 & & & 6.00 & & 15.70 & & \\
\hline
\end{tabular}

Donde $\bar{x}$, media; s, desviación estádar; Pi, parámetros; Mi, puntos lóticos; Li, puntos lénticos.

En cuanto a la $\mathrm{DBO}_{5}(\mathrm{P} 2)$, los ríos que atraviesan zonas urbanas reciben efluentes domésticos, industriales y ganaderas normalmente presentan altos valores (Chung, Li, \& Chen, 2005; Amado et al., 2006; Bhatti \& Latif, 2011); este comportamiento es característico para el río Chumbao (Tabla 4), incrementándose el DBO debido a la presencia de materia orgánica, así como a la pendiente pronunciada del río, lo que mejora la oxigenación (Soon \& Seok, 2002). 
Los niveles de nitratos (P3) y fosfatos (P4) son bajos en todos los puntos de muestreo, reportándose pequeños incrementos a lo largo del recorrido del río ( $p$-value $<0.05$ ); ello debido al uso de fertilizantes derivados de nitrato (Tully, Lawrence, \& Scanlon, 2012), y a la basura que se arroja al río que es fuente de fosfatos (Cieszynska, Wesolowski, Bartoszewicz, Michalska, \& Nowacki, 2012), mientras que en las lagunas no se observa contenido de estas sustancias.

En relación con la temperatura (P5), se incrementa aguas abajo de $10.99 \pm 0.11^{\circ} \mathrm{C}$ en $\mathrm{M} 1$ a $15.86 \pm 0.24^{\circ} \mathrm{C}$ en M8 ( $p$-value < 0.05$)$; este hecho se debe al cambio del piso altitudinal y al tiempo de muestreo (Rubio-Arias et al., 2017; Gamarra-Torres et al., 2018); mientras que la temperatura del agua en las lagunas L1 y L2 es menor que en los puntos lóticos, lo cual se debe al piso altitudinal y represamiento (Webb, Hannah, Dan-Moore, Brown, \& Nobilis, 2008; Arbat-Bofill et al., 2014); esta variable tiene influencia directa sobre la composición química del agua y el OD (Rubio-Arias, Contreras-Carveo, Quintana, Saucedo-Teran, \& Pinales-Munguia, 2012).

La turbidez (P6) y los STD (P7) se incrementan aguas abajo, este hecho se debe en gran medida a la erosión natural en temporada de avenidas (Ospina-Zúñiga, García-Cobas, Gordillo-Rivera, \& TovarHernández, 2016), así como a la actividad antrópica, sobre todo por desechos domésticos inorgánicos u orgánicos (Almeida \& Schwarzbold, 2003); Montoya, Loaiza, Torres, Cruz, \& Escobar, 2011), y en gran medida a las actividades extractivas de materiales de construcción civil 
que ocasionan problemas en el hábitat acuático y cambios del régimen hidráulico de la corriente debido a la sedimentación causada por el exceso de sólidos sedimentables (Caruso, 2002; Buzelli, Bianchessi, \& CunhaSantino, 2013; Choque-Quispe et al., 2020).

En referencia al color (P8), no se observa incremento significativo aguas abajo y es ligeramente mayor en las zonas urbanas; esto se puede deber a que el color está relacionado con la presencia de sustancias químicas y tóxicas de los desechos domésticos; sin embargo, en las zonas no urbanas, el color se asocia con sustancias como taninos y ácidos húmicos propios de la vegetación nativa (Camargo-Valero \& Cruz-Torres, 1999).

La alcalinidad (P9) y dureza (P10) aumentan de forma significativa, disminuyendo la capacidad amortiguadora del agua; sin embargo, los resultados de los puntos de muestreo aguas arriba muestran que la capacidad de neutralizar ácidos es baja, por presentar valores de alcalinidad bajos y son susceptibles de acidificarse.

Por otra parte, los valores de $\mathrm{pH}$ (P11) aumentan ligeramente aguas abajo; esta variación se debe sobre todo a la actividad antrópica y sustancias alóctonas y autóctonas que se encuentran en el cauce del río. Este hecho hace que la conductividad (P12) y la concentración de cloruros (P13) se incrementen fuertemente sobre todo en las zonas urbanas (M5, M6, M7 Y M8) debido a la erosión causada por el agua de riego (Tebbutt, 1998) de los campos de cultivos en las zonas altas (M1, M2 y M3); no 
obstante, el comportamiento de estos parámetros depende de depósitos de sales y tipos de suelos en las áreas aledañas (Páez-Sánchez, AlfaroCuevas, Cortés-Martínez, \& Segovia, 2013).

La carga microbiana se aprecia en dos tramos definidos. Una zona (M1, M2 y M3) con presencia de campos de cultivo y áreas de fauna y flora silvestre, donde los niveles de E. coli (P14) y coliformes totales (P15) son casi nulos. Otra zona, urbana (M4 en adelante), donde los niveles de P14 y P15 se incrementan drásticamente por la presencia de efluentes domésticos y debido a la actividad pecuaria cuyos residuos como est iércol y purines aumentan los niveles de los coliformes (Olsen, Chappell, \& Loftis, 2012; Valenzuela, Godoy, Almonacid, \& Barrientos, 2012).

En cuanto a la calidad del agua, el índice Dinius considera los puntos M1 al M5 como excelente (Figura 5), mientras que los puntos M7 y M8 se refieren como excesivamente contaminados, presentando niveles altos de riesgo sanitario (Torres, Cruz, \& Patiño, 2009), pudiendo causar enfermedades de origen hídrico al hombre y animales, siendo un comportamiento característico de ríos que atraviesan urbes (De Maria \& Moreira, 2007; Rodríguez-Gil, Cáceres, Dafouza, \& Valcárcel, 2018). 
2021, Instituto Mexicano de Tecnología del Agua

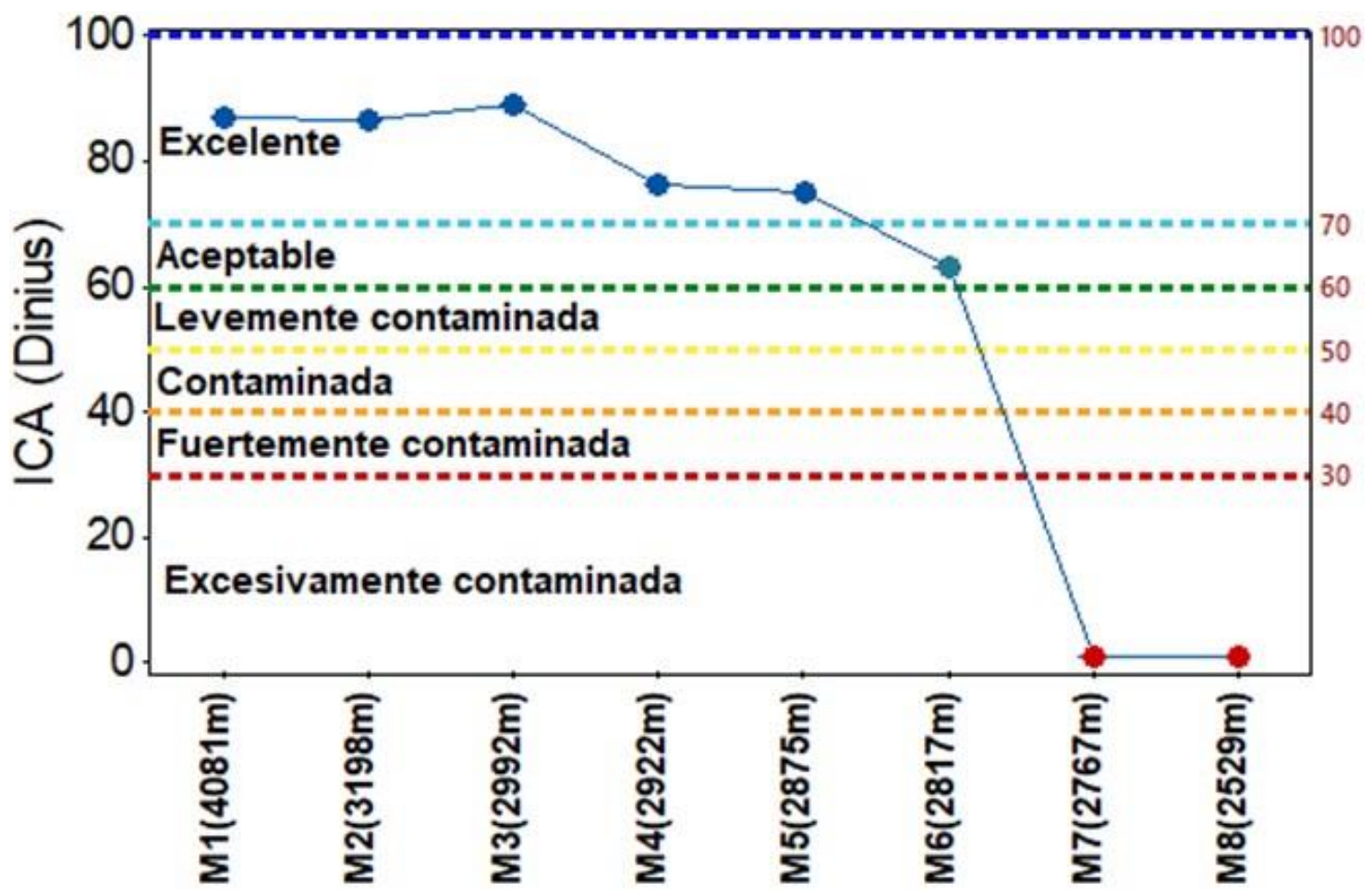

Figura 5. Variación del ICA Dinius por puntos de muestreo.

La temperatura y el OD están relacionados con las condiciones geográficas de la zona de estudio (Hussein \& Ali, 2017) y muestran correlación significativa (> 0.7) (Tabla 5) por la disminución de altitud de 4081 a 2529 m. Del mismo modo, la $\mathrm{DBO}_{5}$ presenta buena correlación con la mayoría de los parámetros en estudio, excepto color y cloruros; esto se debe a que el río Chumbao recibe descarga de materia orgánica residual sólida y líquida, incrementándose los niveles de nitratos, fosfatos, temperatura, turbidez, STD, alcalinidad, dureza, $\mathrm{pH}$, conductividad, que 
Tecnología y

Ciencias $₫$ Agua
2021, Instituto Mexicano de Tecnología del Agua

Open Access bajo la licencia CC BY-NC-SA 4.0

(https://creativecommons.org/licenses/by-nc-sa/4.0/)

muestran alta correlación entre ellos (Tabla 5); ello se evidencia más aún por el incremento del nivel de coliformes a lo largo del recorrido del río, evidenciándose contaminación por actividades antrópicas (Vieira et al., 2012; Peeler, Opsahl, \& Chanton, 2006; Linden et al., 2015), que es característico de ríos que atraviesan zonas urbanas (Cotorro, Gamarra, \& Barboza, 2018; Dhawde et al., 2018; Abbas \& Hassan, 2018).

Tabla 5. Correlación de parámetros e ICA.

\begin{tabular}{|c|c|c|c|c|c|c|c|c|c|c|c|c|c|c|c|}
\hline & P1 & P2 & P3 & P4 & P5 & P6 & P7 & P8 & P9 & P10 & P11 & P12 & P13 & P14 & P15 \\
\hline P2 & 0.47 & & & & & & & & & & & & & & \\
\hline P3 & 0.55 & 0.90 & & & & & & & & & & & & & \\
\hline P4 & 0.31 & 0.92 & 0.80 & & & & & & & & & & & & \\
\hline P5 & 0.70 & 0.86 & 0.78 & 0.70 & & & & & & & & & & & \\
\hline P6 & 0.46 & 0.92 & 0.98 & 0.89 & 0.75 & & & & & & & & & & \\
\hline P7 & 0.63 & 0.94 & 0.98 & 0.85 & 0.85 & 0.97 & & & & & & & & & \\
\hline P8 & 0.61 & 0.31 & 0.01 & 0.40 & 0.02 & 0.15 & 0.02 & & & & & & & & \\
\hline P9 & 0.54 & 0.92 & 0.97 & 0.88 & 0.77 & 0.97 & 0.98 & 0.05 & & & & & & & \\
\hline P10 & 0.29 & 0.93 & 0.83 & 0.92 & 0.82 & 0.89 & 0.85 & 0.43 & 0.85 & & & & & & \\
\hline P11 & 0.57 & 0.87 & 0.80 & 0.65 & 0.91 & 0.76 & 0.84 & 0.17 & 0.75 & 0.79 & & & & & \\
\hline P12 & 0.62 & 0.95 & 0.98 & 0.85 & 0.85 & 0.97 & 1.00 & 0.03 & 0.98 & 0.86 & 0.84 & & & & \\
\hline
\end{tabular}


Tecnología y

Ciencias $₫$ Agua
2021, Instituto Mexicano de Tecnología del Agua

Open Access bajo la licencia CC BY-NC-SA 4.0

(https://creativecommons.org/licenses/by-nc-sa/4.0/)
P13
0.20
$-0.16$
0.13
$0.00 \quad-0.49$
0.14
0.02
0.18
$\begin{array}{llll}0.14 & -0.21 & -0.36 & 0.02\end{array}$
P14 0.36
0.95
0.85
0.99
0.76
0.93
$0.89 \quad 0.37$
0.92
0.95
0.72
0.89
0.03
$\begin{array}{llll}\mathbf{P 1 5} & 0.17 & 0.92 & 0.77\end{array}$
0.87
0.76
0.82
$0.80 \quad 0.51$
0.80
0.94
0.76
0.81
0.24
0.89
ICA
0.48
$\begin{array}{llllll}-0.93 & -0.90 & -0.97 & -0.76 & -0.95 & -0.94\end{array}$
0.20
$-0.96-0.89-0.72-0.93$
0.08
$-0.98-0.81$

Los resultados están calculados a un nivel de significancia del 5 \%.

Por otra parte, se observa que el ICA muestra correlación significativa negativa con los parámetros estudiados $(>0.72)$, excepto con OD (P1), color (P8) y cloruros (P13) (Tabla 5), por lo que cualquier variación de estos parámetros fuera de los límites establecidos en los ECA (MINAM, 2017) permitiría conocer de manera indirecta la calidad del agua del río, y eventualmente disminuir su valor, tal como lo consideran González, Caicedo y Aguirre (2013), y Quiroz, Izquierdo y Menéndez (2017).

\section{Conclusiones}


2021, Instituto Mexicano de Tecnología del Agua

El índice de calidad del agua (ICA) del río Chumbao está distribuido en tres categorías bien definidas: excelente (M1, M2, M3, M4 Y M5) cercanos a la cabecera de la microcuenca y los puntos lénticos (lagunas de Pampahuasi y Paccoccocha); aceptable (M6), y excesivamente contaminado, los puntos M7 y M8 fuera de la zona urbana aguas abajo, pudiendo disminuir ante cualquier cambio de los parámetros en estudio a valores fuera de los ECA.

\section{Agradecimientos}

A la Vicepresidencia de Investigación de la Universidad Nacional José María Arguedas, por el financiamiento parcial de la investigación.

\section{Referencias}

Abbas, A. A. A., \& Hassan, F. M. (2018). Water quality assessment of Euphrates River in Gadisiyah province (Diwaniyah River), Iraq. Iraqi Journal of Agricultural Sciences, 49(2), 251-261. 
Tecnología y

Ciencias $\stackrel{\Xi}{\unlhd}$ gua
2021, Instituto Mexicano de Tecnología del Agua

Open Access bajola licenciaCC BY-NC-SA 4.0

(https://creativecommons.org/licenses/by-nc-sa/4.0/)

Aguirre-Martínez, G. V., André, C., Gagné, F., \& Martín-Díaz, L. M. (2018). The effects of human drugs in Corbicula fluminea. Assessment of neurotoxicity, inflammation, gametogenic activity, and energy status. Ecotoxicology and Environmental Safety, 148, 652-663. DOI: $10.1016 /$ j.ecoenv.2017.09.042.

Almeida, M. A. B., \& Schwarzbold, A. (2003). Avaliação sazonal da qualidade das águas do Arroio da Cria Montenegro, RS com aplicação de um índice de qualidade de água (IQA). Revista Brasileira de Recursos Hídricos, 8(1), 81-97. DOI: 10.21168/rbrh

Amado, J., Rubiños-Panta, J. E., Gavi-Reyes, F., Alarcón-Cabañero, J. J., Hernández-Acosta, E. , Ramírez-Ayala, C., Mejía-Saenz, E., PedreroSalcedo, F., Nicolas-Nicolas, E., \& Salazar-Sosa, E. (2006). Water quality in the basin of the Amajac River, Hidalgo, Mexico: Diagnosis and prediction. Phyton, 75, 71-83. Recuperado de http://www.scielo.org.ar/scielo.php?pid=S1851$56572006000100007 \&$ script=sci_abstract\&tlng=en

APHA, American Public Health Association. (2012). Standard methods for the examination of water and wastewater ( $22^{\text {nd }}$ ed.). Washington, DC, USA: American Public Health Association (APHA), American Water Works Association (AWWA) and Water Environment Federation (WEF).

Arbat-Bofill, M., Sánchez-Juny, M., Bladé, E., Niñerola, D., Dolz, J., \& Palau, A. (2014). Hydrodynamics of Ribarroja Reservoir (Ebro River, 
Spain): Water temperature, water velocities and water age. En: Anton, J. S., Giovanni, C., Mario, J. F., \& Michael, P. (eds.). Proceeding of the International Conference on Fluvial Hydraulics (pp. 1737-1744). Lausanne, Switzerland: Taylor \& Francis Group.

Benvenuti, T., Kieling-Rubio, M. A., Klauck, C. R., \& Rodrigues, M. A. S. (2015). Evaluation of water quality at the source of streams of the Sinos River Basin, southern Brazil. Brazilian Journal of Biology, 75(2), 98-104. DOI: 10.1590/1519-6984.1513.

Bharti, N., \& Katyal, D. (2011). Water quality indices used for surface water vulnerability assessment. International Journal of Environmental Sciences, 2(1), 154-173.

Bhatti, M. T., \& Latif, M. (2011). Assessment of water quality of a river using an indexing approach during the low-flow season. Irrigation and Drainage, 60(1), 103-114. DOI: 10.1002/ird.549

Blume, K. K., Macedo, J. C., Meneguzzi, A., Silva, L. B., Quevedo, D. M., \& Rodrigues, M. A. S. (2010). Waterquality assessment of the Sinos River, Southern Brazil. Brazilian Journal of Biology, 70(4), 11851193. DOI: $10.1590 /$ S1519-69842010000600008

Brown, R. M., Macclelland, N. I., Deininger, R. A., \& Tozer, R. G. (1970). Water Quality Index Do We Dare? Water \& Sewage Works, 117(10), 339-343. 
Buzelli, G., Bianchessi D., \& Cunha-Santino, M. (2013). Análise e diagnóstico da qualidade da água e estado trófico do reservatório de Barra Bonita. SP. Ambiente \& Água-An Interdisciplinary Journal of Applied Science, 8(1), 186-205. DOI: 10.4136/ambi-agua.930

Camargo-Valero, M., \& Cruz-Torres, L. E. (1999). Sustancias húmicas en aguas para abastecimiento. Revista Ingeniería e Investigación, (44), 63-72.

Caruso, B. S. (2002). Temporal and spatial patterns of extreme low flows and effects on stream ecosystem in Otago, New Zealand. Journal of Hydrology, 257(1-4), 115-133. DOI: 10.1016/S00221694(01)00546-7

Choque-Quispe, D., Ligarda-Samanez, C. A., Ramos-Pacheco, B. S., Solano-Reynoso, A. M., Choque-Quispe, Y., Peralta-Guevara, D. E., \& Quispe-Quispe, Y. (2020). Optimization of the flocculating capacity of natural coagulants in water treatment. DYNA, 87(212), 90-95. DOI: $10.15446 /$ dyna.v87n212.80467

Chung, Y. C., Li, Y. H., \& Chen, C. C. (2005). Pollutant removal from aquaculture wastewater using the biopolymer chitosan at different molecular weights. Journal of Environmental Science and Health, 40(9), 1775-1790. DOI: 10.1081/ESE-200068058

Cieszynska, M., Wesolowski, M., Bartoszewicz, M., Michalska, M., \& Nowacki, J. (2012). Application of physicochemical data for water- 
quality assessment of watercourses in the Gdansk Municipality (South Baltic coast). Environmental monitoring and Assessment, 184(4), 2017-2029. DOI: 10.1007/s10661-011-2096-5

Cotorro, F., Gamarra, O., \& Barboza, E. (2018). Evaluación multivariante de la calidad del agua en la cuenca del Utcubamba (Perú). Tecnología y ciencias del agua, 9(5), 33-57. DOI: 10.24850/j-tyca2018-05-02

De Maria, C. A. B., \& Moreira, R. F. A. (2007). Cafeína: revisão sobre métodos de análise. Química Nova, 30(1), 99-105. DOI: $10.1590 /$ S0100-40422007000100021

Dhawde, R., Surve, N., Macaden, R., Wennberg, A. C., Seifert-Dähnn, I., Ghadge, A., \& Birdi, T. (2018). Physicochemical and bacteriological analysis of water quality in drought prone areas of Pune and Satara districts of Maharashtra, India. Environments, 61(5), 1-20. DOI: 10.3390/environments5050061

Dinius, S. H. (1987). Design of an Index of Water Quality. Water Resources Bulletin, 23(11), 833-843.

Feria-Díaz, J. J., Náder-Salgado, D., \& Meza-Pérez, S. J. (2017). Deoxygenation and re-aeration rates of the Sinu River. Ingeniería $y$ Desarrollo, 35(1), 1-17. DOI: 10.14482/inde.35.1.8940 
Ciencias $₫$ Agua
2021, Instituto Mexicano de Tecnología del Agua

Open Access bajo la licencia CC BY-NC-SA 4.0

(https://creativecommons.org/licenses/by-nc-sa/4.0/)

Froehner, S., Machado, K. S., Botelho, D. , \& Cordova-Da-Rosa, E. (2010). Tracking anthropogenic inputs biomarkers in Barigui River-Brazil. J. Water, Air, \& Soil Pollution, 210(1), 33-41. DOI: 10.1007/s11270009-0220-8

Gamarra-Torres, O. A., Barrena-Gurbillón, M. A., Barboza-Castillo, E., Rascón-Barrios, J., Corroto, F., \& Taramona-Ruiz, L. A. (2018). Fuentes de contaminación estacionales en la cuenca del río Utcubamba, región Amazonas, Perú. Arnaldoa, 25(1) 179-194. DOI: $10.22497 /$ arnaldoa.251.25111.

González, V., Caicedo, O., \& Aguirre, N. (2013). Aplicación de los índices de calidad de agua NSF, DINIUS y BMWP. Gestión y Ambiente, 16(1), 97-108.

Horton, R. K. (1965). An index-number system for rating water quality. Journal of the Water Pollution Control Federation, 37(3), 300-306.

Hussein, S., \& Ali, S. (2017). Water quality assessment of Al-Gharraf River, South of Iraq using multivariate statistical techniques. Journal of Al-Nahrain University, 20(2), 114-122. DOI: 10.22401/JUNS.20.2.16

Jacobsen, D., \& Brodersen, K. P. (2008). Are altitudinal limits of equatorial stream insects reflected in their respiratory performance? Freshwater Biology, 53(11), 2295-2308. DOI: 10.1111/j.13652427.2008.02050.x 
Linden, R., Antunes, M. V., Heinzelmann, L. S., Fleck, J. D., Staggemeier, R., Fabres, R. B., Vecchia, A. D., Nascimento, C. A., \& Spilki, F. R. (2015). Caffeine as an indicator of human fecal contamination in the Sinos River: A preliminary study. Brazilian Journal of Biology, 75(2), 81-84. DOI: 10.1590/1519-6984.0513

MINAM, Ministerio del Ambiente. (2017). Estándares de Calidad Ambiental (ECA), Decreto Supremo No 004-2017-MINAM-Perú (10 pp.). Magdalena del Mar, Perú: Ministerio del Ambiente.

Montoya, C., Loaiza, D., Torres, P., Cruz, C. H., \& Escobar, J. C. (2011). Efecto del incremento en la turbiedad del agua cruda sobre la eficiencia de procesos convencionales de potabilización. Revista $E I A, 16,137-148$.

Mophin-Kani, K., \& Murugesan, A. G. (2011). Evaluation and classification of water quality of perennial river Tamirabarani through aggregation of water quality index. International Journal of Environmental Protection, 1(5), 24-33. DOI: 10.5963/IJEP0105004

Olsen, R. L., Chappell, R. W., \& Loftis, J. C. (2012). Water quality sample collection, data treatment and results presentation for principal components analysis-literature review and Illinois River watershed case study. Water Research, 46(9), DOI: 3110-3122. $10.1016 /$ j.watres.2012.03.028 
Tecnología y

Ciencias $\stackrel{\unlhd}{\unlhd}$ gua
2021, Instituto Mexicano de Tecnología del Agua

Open Access bajola licencia CC BY-NC-SA 4.0

(https://creativecommons.org/licenses/by-nc-sa/4.0/)

Ospina-Zúñiga, O., García-Cobas, G., Gordillo-Rivera, J., \& TovarHernández, K. (2016). Evaluación de la turbiedad y la conductividad ocurrida en temporada seca y de lluvia en el río Combeima (Ibagué, Colombia). Ingeniería Solidaria, 12(19), 19-36. DOI: 10.16925/in.v12i19.1191

Páez-Sánchez, A., Alfaro-Cuevas, R., Cortés-Martínez, R., \& Segovia, N. (2013). Arsenic content and physicochemical parameters of water from wells and thermal springs at Cuitzeo Lake Basin, Mexico. International Journal of Innovative Research in Science, Engineering and Technology, 2(12), 7731-7740.

Peeler, K. A., Opsahl, S. P., \& Chanton, J. P. (2006). Tracking anthropogenic inputs using caffeine, indicator bacteria, and nutrients in rural freshwater and urban marine systems. Environmental Science \& Technology, 40(24), 7616-7622. DOI: $10.1021 /$ es061213c

Prakirake, C., Chaiprasert, P., \& Tripetchkul, S. (2009). Development of specific water quality index for water supply in Thailand. Songklanakarin Journal of Science and Technology, 31(1), 91-104.

Quiroz, L. S., Izquierdo, E., \& Menéndez, E. (2017). Aplicación del índice de calidad de agua en el río Portoviejo, Ecuador. Ingeniería Hidráulica y Ambiental, 38(3), 41-51. 
Rivera, J. V. (2011). Evaluation of organic matter in the cold river supported in QUAL2K version 2.07. DYNA, 78(169), 131-139. Recuperado de http://www.scielo.org.co/scielo.php?script=sci_arttext\&pid=S0012 $-73532011000500015$

Rodríguez-Gil, J. L., Cáceres, N., Dafouza, R., \& Valcárcel, Y. (2018). Caffeine and paraxanthine in aquatic systems: Global exposure distributions and probabilistic risk assessment. Science of the Total Environment, 612, 1058-1071. DOI: 10.1016/j.scitotenv.2017.08.066

Rubio-Arias, H., Contreras-Carveo, M., Quintana, M., Saucedo-Teran, R. A., \& Pinales-Munguia, A. P. (2012). An Overall WaterQuality Index (WQI) for a Man-Made Aquatic Reservoir in Mexico. International Journal of Environmental Research and Public Health, 9(5), 16871698. DOI: $10.3390 /$ ijerph9051687

Rubio-Arias, H., Ochoa-Rivero, J. M., Ortiz-Delgado, R. C., Quintana, R. M., Saucedo-Terán, R. A., \& Villalba, M.L. (2017). Water quality using physical-chemical and metal parameters in three contrasting locations of the Conchos river in Chihuahua, Mexico. Investigación y Ciencia, 25(70), 13-22. Recuperado de https://www.redalyc.org/articulo.oa?id=67451351002 
Sanders, N. J. (2002). Elevational gradients in ant species richness: Area, geometry and Rapoport's rule. Ecography, 25(1), 25-32. DOI: 10.1034/j.1600-0587.2002.250104.x

Soon, S., \& Seok, Y. (2002). A water quality modeling study of the Nakdong River, Korea. Ecological Modelling, 152(1) 65-75. DOI: 10.1016/S0304-3800(01)00489-6

Tebbutt, T. H. Y. (1998). Principles of waterquality control ( $5^{\text {th }}$ ed.). DOI: 10.1016/B978-0-7506-3658-2.X5000-9

Torres, P., Cruz, C., \& Patiño, P. (2009). Índices de calidad de agua en fuentes superficiales utilizadas en la producción de agua para consumo humano. Una revisión crítica. Revista Ingenierías Universidad de Medellín, 8(15), 79-94. Recuperado de http://www.scielo.org.co/pdf/rium/v8n15s1/v8n15s1a09.pdf

Torres, P., Cruz, C., Patiño, P., Escobar, J. C., \& Pérez, A. (2010). Applying water quality indexes (WQI) to the use of water sources for human consumption. Ingeniería e Investigación, 30(3), 86-95. Recuperado de http://www.scielo.org.co/pdf/iei/v30n3/v30n3a07.pdf

Tully, K. L., Lawrence, D., \& Scanlon, T. M. (2012). More trees less loss: Nitrogen leaching losses decrease with increasing biomass in coffee agroforests. Agriculture, Ecosystems and Environment, 161, 137144. DOI: $10.1016 / \mathrm{j}$.agee.2012.08.002. 
2021, Instituto Mexicano de Tecnología del Agua

Tecnología y

Open Access bajo la licencia CC BY-NC-SA 4.0

(https://creativecommons.org/licenses/by-nc-sa/4.0/)

Valenzuela, E., Godoy, R., Almonacid, L., \& Barrientos, M. (2012). Calidad microbiológica del agua de un área agrícola-ganadera del centro sur de Chile y su posible implicancia en la salud humana. Revista Chilena de Infectología, 29(6), 628-634. DOI: 10.4067/S071610182012000700007

Vieira, J. S., Pires, J. C., Martins, F. G., Vilar, V. J., Boaventura, R. A., \& Botelho, C. M. (2012). Surface waterquality assessment of Lis River using multivariate statistical methods. Water, Air, \& Soil Pollution, 223(9), 5549-5561. DOI: 10.1007/s11270-012-12675

Webb, B. W., Hannah, D. M., Dan-Moore, R., Brown, L. E., \& Nobilis, F. (2008). Recent advances in stream and river temperature research. Hydrological Processes, 22(7), 902-918. DOI: 10.1002/hyp.6994 